Chapter 3

\title{
Progress in Polymer Thermoelectrics
}

\author{
Lukas Stepien, Aljoscha Roch, Roman Tkachov and \\ Tomasz Gedrange
}

Additional information is available at the end of the chapter

http://dx.doi.org/10.5772/66196

\begin{abstract}
This chapter addresses recent progress in the field of polymer thermoelectric materials. It covers a brief introduction to intrinsically conductive polymers and its motivation for thermoelectric utilization. A review about important and recent literature in the field of p-type and n-type polymers for thermoelectric applications is summarized here. For a better understanding of material development issues, doping mechanisms for intrinsically conducting polymers are discussed. Special emphasis is given to n-type polymers, since this group of polymers is often neglected due to unavailability or poor stability during processing. Different possibilities in terms of generator design and fabrication are presented. Recent challenges in this scientific field are discussed in respect to current material development, uncertainty during the measurement of thermoelectric properties as well as temperature stability for the most prominent p-type polymer used for thermoelectric, PEDOT:PSS.
\end{abstract}

Keywords: printing, coating, intrinsically conductive polymers, PEDOT:PSS, flexible, thermoelectric generator

\section{Introduction}

Historically, the most famous intrinsically conducting polymer is polyacetylene, which was discovered in 1976 by Alan Heeger, Alan MacDiarmid, and Hideki Shirakawa, who were jointly awarded with Nobel Prize in the year 2000. After the discovery that doping (in the case of polyacetylene it is chemical oxidation with iodine) of polymer chains can increase electrical conductivity of the polymer dramatically, a lot of effort was put in investigating the doping process as well as polymer synthesis itself [1]. 
In contrast to known solid state semiconductors, polymers can be doped by different approaches following the concept of MacDiarmid on primary and secondary doping. Primary doping includes chemical doping mechanism, which can be oxidizing/reducing or protonating/deprotonating. Secondary doping addresses the change in the polymer morphology like chain alignment, chain orientation, crystallinity, and so on.

However, for a reasonable utilization of these electrical conducting polymers, good processability and stability are the major requirements. This leads to considerable amount of different intrinsically conducting polymers, e.g., polyaniline, polycarbazole, and polythiopene.

In recent years, significant progress in the development of new types of conductive polymers has been achieved. Plenty of scientific publications can be found in scientific journals for polymer synthesis, polymer modification, or characterization.

For thermoelectric applications, both types of conducting polymers were investigated, on the one hand, pure organic polymers such as PANI [2], PPV [3], PPy:Tos [4], PEDOT:PSS [5], or PEDOT: PEDOT:TOS [6, 7], but on the other hand, metal organic complexes [8] as well as composites with nanostructures. The pure organic polymers are generally semiconductors and need to be primary doped in order to become electrically conductive. The oxidation leads to p-type conductivity. The degree of oxidation determines charge carriers' concentration and, therefore, affects the electrical conductivity directly. Electrical conductivity of p-type polymer like PEDOT:PSS with high degree of oxidation can reach up to $2000 \mathrm{~S} / \mathrm{cm}$ [9] and even $3300 \mathrm{~S} / \mathrm{cm}$ [10]. It was reported recently [11] about reaching electrical conductivity up to $4600 \mathrm{~S} / \mathrm{cm}$ by active control of the deposition procedure of polymer PEDOT:PSS. It was possible to achieve conductivity up to $5400 \mathrm{~S} / \mathrm{cm}$ by changing the counter ions of PEDOT [12]. Vapor-phase-grown single crystal PEDOT nanowires showed electrical conductivity of 8797 $\mathrm{S} / \mathrm{cm}$ [13], which is the highest value known for this group of polymers. In spite of this outstanding electrical conductivity, Seebeck coefficient is usually relatively small for polymers.

Among p-type polymers, PEDOT, modified in PEDOT:TOS and PEDOT:PSS, respectively, is the most investigated polymer for thermoelectric utilization. Reported ZT values are in the range 0.2-1.02 for PEDOT:TOS [5-7]. Unfortunately, the reported performances often lack of reproducibility by other working groups.

The synthesis of n-type polymers involves other challenges compared to the development of p-type polymers. Stability of n-type polymer under ambient conditions is often critical, and electrical conductivity is in general not as high as for p-type polymers. However, a synthesis approach with metal organic polymers is promising and it was shown, that ZT value of this material can reach 0.2 [8].

Figure 1 shows overview about last years' progress in ZT values of conductive polymers.

\section{Intrinsically conductive polymers for thermoelectric application}

This section gives an overview of the recent advances made in polymer thermoelectric materials, which covers different material classes. First, considerations regarding the benefits of polymers for thermoelectric applications are discussed. Second, short introduction to doping 
of intrinsically conductive polymers is given. Subsequently, overview of p-type and n-type polymers is presented.

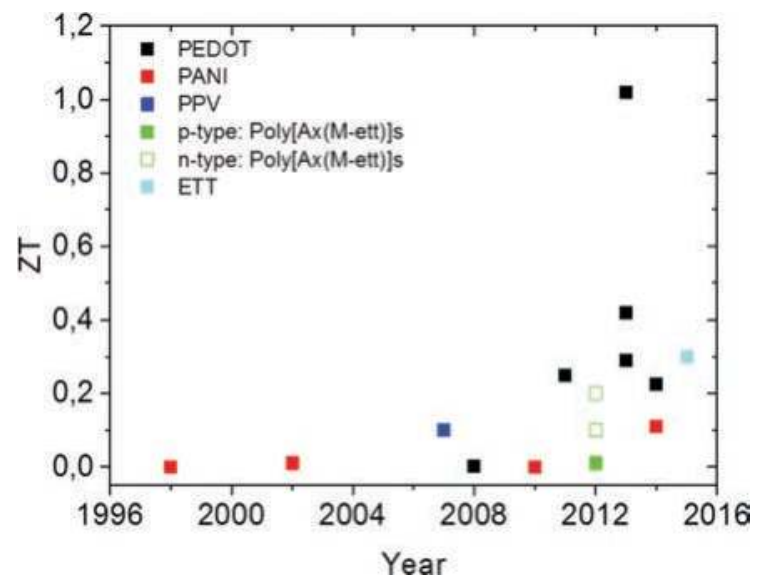

Figure 1. Overview of ZT values of conductive polymers, which were published recently $[2,3,5-8,14-16]$.

The main requirement for a polymer to be electrically conductive is the formation of a conjugated pi-electron system. With the increase of the delocalized pi-electron system, the polymer changes its nature from an isolator to a semiconductor [17]. The band gap of intrinsically conductive polymers can vary from $1.36 \mathrm{eV}$ (for polyacetylene) to $5.96 \mathrm{eV}$ (for polypyrrole) [18]. In order to increase electrical conductivity of these semiconductive polymers, one has to increase the free charge carriers concentration (analogy to silicon-based semiconductors). This doping leads to the formation of additional energy levels (allowed energy levels) within the band gap. Due to these new energy levels, free charge carriers can arise, which can be dislocated along the polymer chain or hop/tunnel between individual polymer chains. A brief introduction can be found in reference [19].

In the beginning, conductive polymers were investigated for thermoelectric applications, especially because of their alleged low thermal conductivity and high electrical conductivity. Low thermal conductivity would make them attractive for producing advanced thermoelectric materials. For technical polymers like PVC or PMMA, thermal conductivity is in the range $0.13-0.3 \mathrm{~W} /(\mathrm{m} \mathrm{K})$ [20]. In the case of intrinsically conductive polymers, thermal conductivity is higher due to the contribution to heat transport by charge carriers. In the case of drop casted films of PEDOT:PSS, thermal conductivity can vary from 0.29 to $1 \mathrm{~W} /(\mathrm{m} \mathrm{K})$ for cross-plane and in-plane measurements, respectively. It was also shown, that in-plane thermal conductivity of these films increases with electrical conductivity [21]. For comparison, spark plasma sintered BiTe compounds also show a relatively low thermal conductivity of $0.8 \mathrm{~W} /(\mathrm{m} \mathrm{K})$ for cross-plane and $1.2 \mathrm{~W} /(\mathrm{m} \mathrm{K})$ for in-plane measurements [22]. For nanostructured thermoelectric materials, thermal conductivity can be even lower [23]. This example shows also, the importance of distinguishing between in-plane and cross-plane measurements. This should also be applied to electrical properties measurements. 
True arguments regarding advantages, comparing to other (brittle) thermoelectric materials, are possible flexibility and mostly nontoxic properties. Abundance, independence on volatile raw material costs, feasible scale-up for material production, as well as, efficient processability of polymer material can be considered as advantages for polymer thermoelectric materials. Polymers are processable by different (industrial) established and sophisticated technologies and the final building up of thermoelectric modules based on polymers is possible with printing techniques. This offers cost-effective production of thermoelectric generators (TEG) at industrial scale.

To facilitate the usage of intrinsically conductive polymers for thermoelectric applications, one has to consider several requirements besides, undoubtedly important, thermoelectric properties. These requirements imply costs, abundance, recyclability, environmental stability, thermal stability, processability, and more.

Basic factors that determine thermoelectric properties of polymers are as follows:

- nature of polymer structure itself;

- kind of counterions;

- polymer chain length, structural order; and

- environmental factors like temperature and humidity.

Of course, other factors like charge carriers density and morphology play an important role in terms of thermoelectric properties and will be discussed below.

\subsection{Doping mechanism for intrinsically conductive polymers}

Thermoelectric properties of polymers are predominantly governed by doping. While undoped polymers are considered to be bad conductive semiconductors, doping can change this state and transfer into metallic-like behavior. Following doping concept of MacDiarmid [24], doping of polymers can be subdivided into primary and secondary doping.

In general, primary doping affects charge carriers density in the polymer material, which influences Seebeck coefficient and electrical conductivity directly. The extent of this effect is related to the used doping agent, which chemically affects polymer backbone, hence creating/ reducing number of free charge carriers.

Changes in thermoelectric properties from secondary doping are related to morphological modification of polymer chains (atomic scale) or grains/crystals (nano-to-microscale). Since this modification does not change the chemical environment of the polymer, thus not changing of charge carriers density, no influence on Seebeck coefficient is expected.

Mobile charge carriers can be dislocated over the polymer backbone. However, not only movement of charge carriers along polymer chain, but also from chain to chain can occur. Charge transfer between chains is considered in many models (e.g., variable-range-hopping, Sheng's model). Notably, charge carriers' mobility along the chain and interchain is different. Typically, charge carriers' mobility along chain is higher than for hopping events. From this follows that better chain alignment, or crystallinity, will directly affect 
macroscopic electrical conductivity. Yet, increased orientation will also increase anisotropy of the material.

For instance, electrical conductivity of iodine doped polyacetylene could be increased by one order of magnitude after stretching [25].

\subsection{P-type conductive polymers}

In spite of high electrical conductivity and power factor, polyacetylene plays no role in thermoelectric usage due to poor processability, unstable doping (with iodine), and high reactivity of solitons (charge carriers), leading to decrease in electrical conductivity over time.

The most prominent representatives of p-type polymers are aromatic polymers with polaron conduction mechanism. This can be found in the family of polythiopenes and others. The most promising polymer is currently PEDOT. Other polymers like P3HT or PANI also showed good improvement, however, they cannot reach the outstanding performance of PEDOT yet.

Review of recently reported material properties are given in Table 1. Further data can be found in references [26-32].

\begin{tabular}{|c|c|c|c|}
\hline Material, reference & Conductivity, S/cm & Seebeck coefficient, $\mu \mathrm{V} / \mathrm{K}$ & $\begin{array}{l}\text { Power Factor, } \\
\mu \mathrm{W} /\left(\mathrm{mK}^{2}\right)\end{array}$ \\
\hline PEDOT: TOS [6] & 74 & 210 & 324 \\
\hline PEDOT:PSS [5] & 890 & 72 & 469 \\
\hline PP-PEDOT: TOS [7] & 1320 & 98 & 1270 \\
\hline PEDOT:PSS [33] & 677 & 28 & 55 \\
\hline PEDOT:PSS [33] & 788 & 32 & 83 \\
\hline PEDOT:PSS [34] & 1400 & 19 & 48 \\
\hline PEDOT:PSS [11] & 4800 & - & - \\
\hline PEDOT:Sulf-NMP [12] & 5400 & - & - \\
\hline PEDOT:PSS/rGO [35] & 637 & 26 & 45 \\
\hline PEDOT:PSS-Tellurim [36] & 215 & 115 & 284 \\
\hline P3HT +50 wt. \% CNT [37] & 345 & 97 & 325 \\
\hline P3HT [38] & 225 & 53 & 62 \\
\hline Poly[Cu $\left.{ }_{x}(\mathrm{Cu}-\mathrm{ett})\right][8]$ & 10 & 83 & 7 \\
\hline Poly[Cu $\left.{ }_{x}(\mathrm{Cu}-\mathrm{ett})\right][39]$ & 1 & 45 & 0.1 \\
\hline Polyacetylene [40] & 700 & 17 & - \\
\hline PANI [41] & 10 & 12 & 0.2 \\
\hline PANI + 50 wt. $\%$ GN sheets [41] & 60 & 30 & 8 \\
\hline PANI + SWCNT [42] & 1440 & - & 217 \\
\hline PANI + graphene [43] & 814 & 26 & 55 \\
\hline
\end{tabular}

Table 1. Thermoelectric properties of selected p-type polymer semiconductors. 


\subsection{N-type conductive polymers}

To make complete thermoelectric (TE) module, an n-type TE material is also required. However, the number of n-type organic materials with good thermoelectric properties is much smaller in comparison to that of p-type materials.

The main reasons are difficulties in n-type doping, because, typically, dopants providing one-electron transfer must have low ionization energies, which leads to instability in air. The next reason is the absence of a large variety of intrinsically conductive polymers. But, in the past 5 years, the number of reports, devoted to n-type organic materials, is steadily growing. It was found that promising materials are n-type fullerenes $\mathrm{K}_{\mathrm{r}} \mathrm{C} 70$ [44], fullerenes C60 doped with $\mathrm{Cr}_{2}(\mathrm{hpp})_{4}$ (hpp = 1,3,4,6,7,8-hexahydro-2 H-pyrimido[1, 2-a]pyrimidine) [45], poly[K $\mathrm{K}_{x}\left(\mathrm{Ni}\right.$-ett)] (ett $=1,1,2,2$-ethenetetrathiolate) [8], poly $\left\{\mathrm{N}, \mathrm{N}^{\prime}\right.$-bis(2-octyl-dodecyl)1,4,5,8-napthalene dicarboximide-2,6-diyl]-alt-5,5'-(2,2'-bithiophene)\} P(NDIOD-T2) doped by dihydro-1H-benzimidazole-2-yl (N-DBI) derivatives [46], self-doped perylene diimides (PDI) [47], polyethylenimine (PEI)/diethylenetriamine (DETA)-doped CNT, that were further reduced by $\mathrm{NaBH} 4$ [48], poly(p-phenylene vinylene) derivatives (FBDPPV) doped with (4-(1,3-dimethyl-2,3-dihydro-1H-benzoimidazol-2-yl)phenyl)dimethylamine (N-DMBI) [49], CoCp2@SWNTs [50], three-dimensional copper 7,7,8,8-tetracyanoquinodimethane (CuTCNQ) [51], isoindigo-based conjugated polymers (IIDT) [52], nanostructured tetrathiotetracene (TCNQ)2 (TTT(TCNQ)2) [53], and polyaniline doped with aprotic ionic liquid [54] (Table 2).

\begin{tabular}{|c|c|c|c|}
\hline Material, reference & Conduc-tivity, $\mathrm{S} / \mathrm{cm}$ & $\begin{array}{l}\text { Seebeck coefficient, } \\
\mu \mathrm{V} / \mathrm{K}\end{array}$ & $\begin{array}{l}\text { Power Factor, } \\
\mu \mathrm{W} / \mathrm{mK}^{2}\end{array}$ \\
\hline $\mathrm{K}_{x} \mathrm{C}_{70}[44]$ & 550 & -22.5 & 28 \\
\hline $\mathrm{C}_{6} 0$ doped with $\mathrm{Cr}_{2}(\mathrm{hpp})_{4}[45]$ & 4 & -175 & 12 \\
\hline $\operatorname{poly}\left[\mathrm{K}_{x}(\mathrm{Ni}\right.$-ett $\left.)\right][8]$ & 40 & -122 & 60 \\
\hline P(NDIOD-T2)) doped with N-DBI [46] & 0.008 & -850 & 0.6 \\
\hline PDI [47] & 0.5 & -168 & 1.4 \\
\hline PEI/DETA-doped CNT [48] & 52 & -86 & 38 \\
\hline FBDPPV doped with N-DMBI [49] & 14 & -141 & 28 \\
\hline CoCp2@SWNTs [50] & 432 & -41.8 & 75.4 \\
\hline CuTCNQ [51] & 0.037 & -632 & 1.5 \\
\hline IIDT [52] & $7 \times 10^{-8}$ & -898 & \\
\hline nanostructured TTT(TCNQ)2 [53] & 12000 (asses.) & -150 (asses.) & \\
\hline polyaniline doped with aprotic ionic liquid [54] & 0.0023 & -138.8 & $4.43 \times 10^{-3}$ \\
\hline $\begin{array}{l}\text { poly }\left[\mathrm{K}_{x}(\mathrm{Ni}-\mathrm{ett})\right] \text {, prepared by an electrochemical } \\
\text { method [55] }\end{array}$ & 200 to 400 & -90 to -140 & 453 \\
\hline
\end{tabular}

Table 2. Thermoelectric properties of selected n-type organic semiconductors. 
Taking into account the very recent research of poly[K $\left.\left.\mathrm{K}_{x} \mathrm{Ni-ett}\right)\right]$ [55], it is one of the most promising n-type materials due to its excellent thermoelectric properties. Despite the very simple synthesis (Figure 2), chemical structure of this polymer is still not exhaustively clear, and is currently the research object. However, prototypes of generators based on this polymer have already shown very promising results. So, provided achieving good processability of this polymer, it can become one of main n-type materials for manufacture of TEGs. Below, we describe in a little more detailed manner, the present state-of-the-art in this topic.

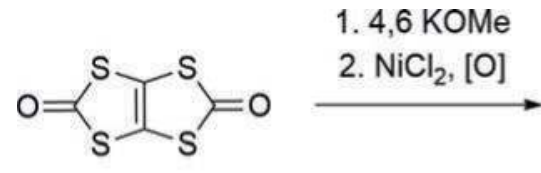

$1, T P D$

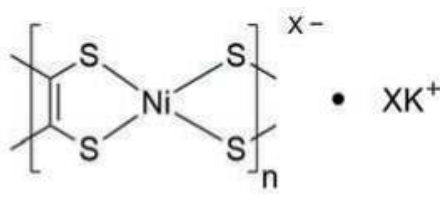

2, Poly[Kx(Ni-ett)]

Figure 2. Synthesis of poly[ $\left.\mathrm{K}_{x}(\mathrm{Ni}-\mathrm{ett})\right]$.

Poly(nickel1,1,2,2-ethenetetrathiolate), poly[ $\mathrm{K}_{\mathrm{x}}(\mathrm{Ni}$-ett) $]$ ( $\mathrm{K}$ is an alkali metal) (Figure 2) as an n-type organic polymer has been already known for a long time [56-64], its thermoelectric properties were carefully evaluated by Zhu's group [8] and an "all-organic" TEG device was fabricated.

Thanks to ambient stability and exciting thermoelectric characteristics, ZT value was equal to 0.2 at $400 \mathrm{~K}$. This inspired other groups to work with this polymer for thermoelectrical applications [65] or to develop its composites with carbon nanotubes [66-68]. One major drawback of poly[ $\left.\mathrm{K}_{x}(\mathrm{Ni}-\mathrm{ett})\right]$ is its poor processability due to its completely insoluble nature leading to suspensions with broad particle size distributions.

Since this polymer is totally insoluble, commonly used structure characterization methods cannot be applied for this polymer, so these compounds have no clearly established structure. Its thermoelectric properties are not completely reproducible, even by the same personnel in the same laboratory. Due to the same reason, it is not possible to control polymerization reaction, for example, to control the degree of polymerization and have an influence on polydispersity of the resulting product. The exact structures of the terminal groups are also not known. Also, because of insolubility, it is not possible to improve properties of polymer after its production-methods like Soxhlet extraction, reprecipitation, or separation of products with the help of column chromatography cannot be applied. The most significant disadvantage of such insolubility and, as a result, bad processability is difficulty for utilization in devices like thermoelectric generators.

To solve the above-mentioned problem, many researchers tried to obtain different composite materials on the basis of these polymers and another compound-poly(vinylidene fluoride) (PVDF) solution [69], 1-butyl(or decyl)-3-methylimidazolium tetrafluoroborate [70], and dodecyltrimethylammonium bromide [71]. It results in great improvement of its processability, but TE properties of such material decrease dramatically, especially the electrical conductivity. 
But, there is also another approach for making paste suitable for printing, which allows avoiding the degradation in thermoelectric performance. It is possible not to modify the polymer itself, but to change the procedure of its preparation (temperature of reaction mixture, speed of rotation, different system of solvents, and the ratio of the solvents, access of oxygen, and so on). Usually, such procedure consists of two sequential stages. First one (preparation of the monomer in fact) is reaction of 1,3,4,6-tetrathiapentalene-2,5-dione (TPD) with potassium methoxide. The second stage (namely, polymerization) is the addition of a metal salt to the solution. Usually $[8,56]$, both steps are carried out in methanol, and both with reflux. After that, the final product is modified to obtain flexible composites [69-71]. But carrying out the polymerization reaction in $\mathrm{N}$-methylformamide (NMF)-methanol medium under certain temperature conditions allows obtaining of the product as a paste with controllable viscosity. Further evaporation of solvent and washing sequentially with water and methanol provide the thermoelectric material with characteristics similar to the powder.

The product of polymerization reaction poly[ $\mathrm{K}_{x}(\mathrm{Ni}$-ett)] (Figure 2) is an alternating copolymer with organic and inorganic monomeric units. The important feature of this reaction is that it occurs without a catalyst. Also, at the moment, it is not possible for the quantitative description of the role of atmospheric oxygen as an oxidizing agent in the polymerization process. Therefore, it is not suitable to describe the rate of reaction with patterns of chain growth or step growth mechanism.

By creating the proper conditions of this synthesis, the produced gel has no fluidity and is stable indefinitely under inert conditions. Even 1 month after the formation and storage under an inert atmosphere, it shows no signs of aging. However, when the gel is placed in the ambient atmosphere, it starts to slowly delaminate, by forming two phases-liquid and precipitate. Obviously, the reason of delamination is oxidation of paste components, leading to further polymerization and formation of insoluble product. It is possible to see very clearly the formation of a gel-like phase and its further separation, accompanied by reduction in viscosity which can be shown by rheological experiments. The diagram (Figure 3) reflects the dependence of the shear viscosity over time in a methanol-N-methylformamide system. During gel formation, a sharp increase in shear viscosity can be noticed and a subsequent reduction of shear viscosity related obviously to delamination of gel during the oxidation process.

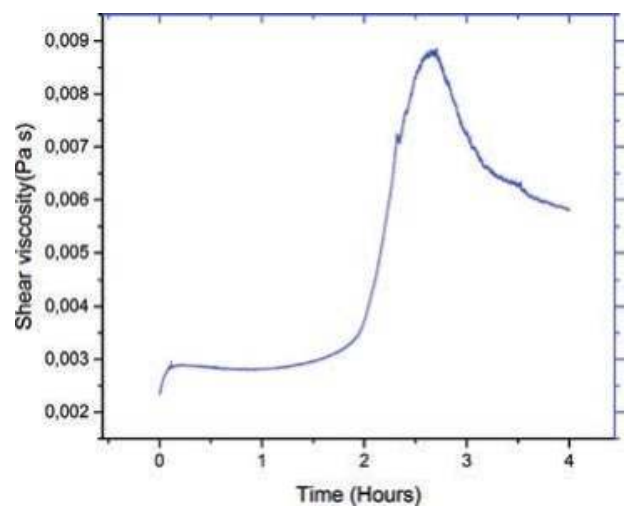

Figure 3. Measurement of shear-viscosity in the ambient atmosphere. 
The film, produced by airbrush-gun, is inhomogeneous and thin $(\sim 763 \mathrm{~nm})$. But it still has a relatively good conductivity $(0,13 \mathrm{~S} / \mathrm{cm}$ (make units used equal)). More homogeneous films are formed by using dispenser printer. Prepared films on Kapton substrate have shown poor adhesion and could be easily removed in water. However, gentle immersion in water kept the film intact and improved its performance $(S=-53.6 \mu \mathrm{V} / \mathrm{K}, \sigma=1.60 \mathrm{~S} / \mathrm{cm})$. Interestingly, it has almost the same thermoelectric characteristics like the powder produced by drying of solvent from paste (see Table 3). Bending test shows, that any appreciable change in conductivity of this film occurs only when winding the tube diameter of less than $4 \mathrm{~mm}$. It is important to note, that in an inert atmosphere thermoelectric properties of polymer are stable.

\begin{tabular}{llll}
\hline Probe, reference & Conductivity, S/cm & Seebeck coefficient, $\mu \mathrm{V} / \mathrm{K}$ & $\begin{array}{l}\text { Power Factor, } \\
\mu W /\left(\mathrm{mK}^{2}\right)\end{array}$ \\
\hline Cuboid, pressed from powder [8] & 40 & -122 & 60 \\
Film [69] & 8.31 & -67.4 & 3.71 \\
Film [71] & 1.14 & -49.0 & 0.28 \\
Tablet pressed from powder [this work] & 1.81 & -44.5 & 0.36 \\
Film made from suspension [this work] & 0.13 & -34.5 & 0.02 \\
Film made from paste [this work] & 1.60 & -53.6 & 0.46 \\
\hline
\end{tabular}

Table 3. Thermoelectric properties of selected poly[ $\left.\mathrm{K}_{x}(\mathrm{Ni}-\mathrm{ett})\right]$.

During first day of exposure in the ambient atmosphere, electrical conductivity of the film, as well as, the pure powder tends to deteriorate. After the fifth day, the thermoelectric properties remained stable for several months (shown in Figure 4).

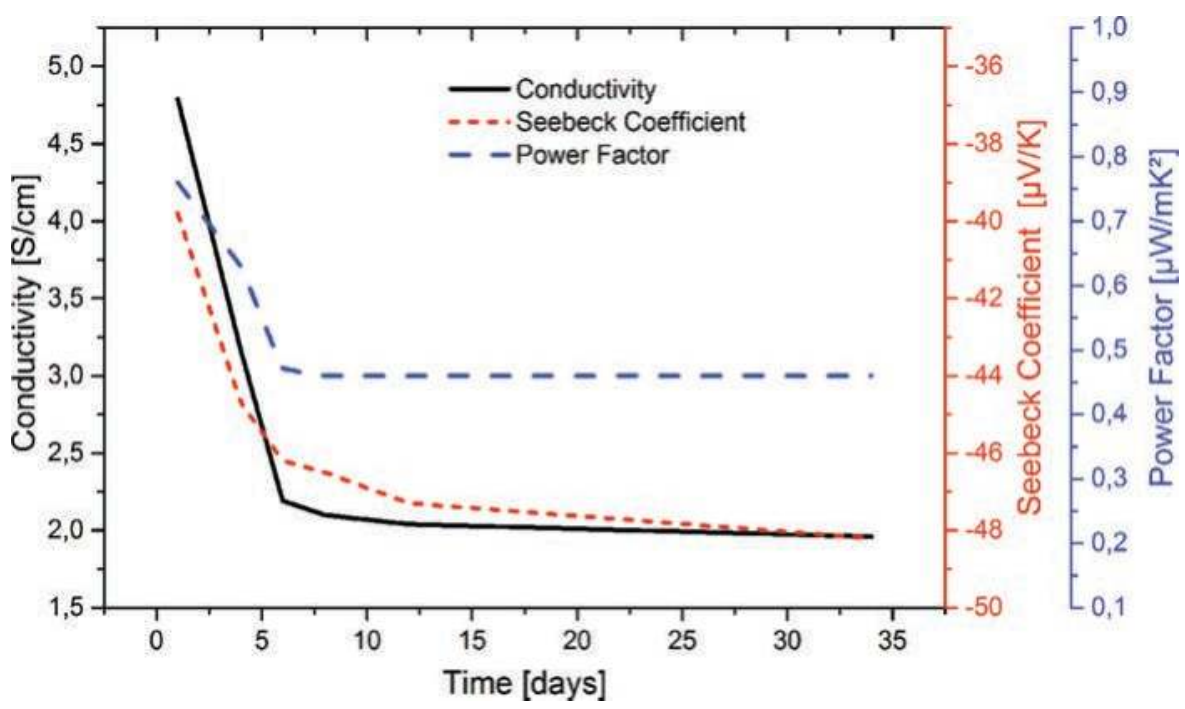

Figure 4. Evolution of thermoelectric properties of Poly $\left[\mathrm{K}_{x}(\mathrm{Ni}-\mathrm{ett})\right]$ under ambient atmosphere. 
To conclude, a simple procedure has been proposed to obtain printable paste based on insoluble conductive polymer poly[ $\left.\mathrm{K}_{x}(\mathrm{Ni}-\mathrm{ett})\right]$. This method opens a way for various applications, such as aerosol and dispenser printing.

\section{Thermoelectric modules}

The performance of polymer TEG is in general lower in comparison to TEGs made by inorganic materials like BiTe. The main reason for this is, of course, the higher thermoelectric performance of BiTe. The ZT value of BiTe is around 1 [22]. ZT values of p-type and n-type polymers are generally clearly below 1 or even below 0.1 .

However, polymers offer other advantages like flexibility or easy processing and fabrication techniques. Most polymers can be printed without losing dramatically electrical and thermoelectric performance. For other materials like BiTe that performance is generally dependent on hot pressing or spark plasma sintering (SPS) techniques followed by cutting which may include manual work or other different processing steps. This advantage of polymers opens opportunities for industrial and economical production of thermoelectric devices.

A well-known technique for printing polymer TEG is the dispense technique. With dispenser, both types of TEGs, mono-leg-TEG (only one polymer), as well as, TEG with p- and n- type polymers can be easily printed, as shown in different publications. Figure 5 shows mono-leg TEG designs.
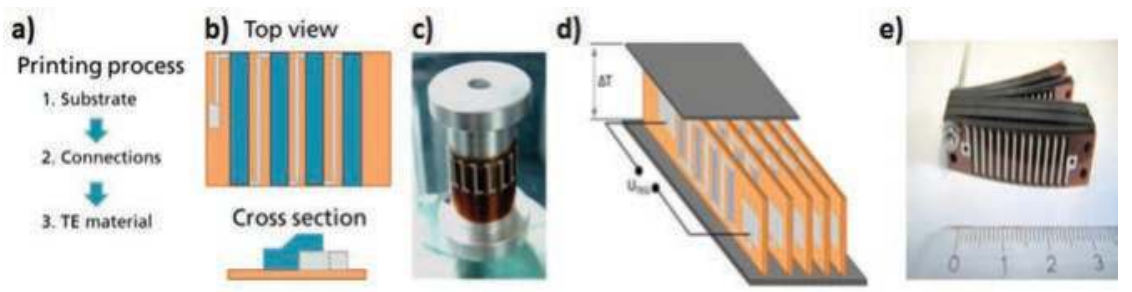

Figure 5. Typical design for printed polymer TEG as mono-leg with one polymer (lateral design). The printing sequence is shown (a). A top view and cross section is displayed (b). In (c), a mono-leg TEG printed with PEDOT:PSS and Ag-paste is shown. TEG is wrapped around holder. (d) Scheme of a stacked mono-leg TEG design. (e) Printed sheets of a mono-leg TEG before assembly.

The same concept is also used for polymer TEG with both p-type and n-type materials. In this case, conductive paste (e.g., silver paste) is replaced by second polymer (see Figure 6). The design concepts shown in Figures 5 and $\mathbf{6}$ have a disadvantage consisting in the fact that heat flow is parallel to the substrate and, therefore, parasitic heat flows exist through the substrate.

Furthermore, thermal connection to a heat source and a heat sink is relatively complex and difficult. Figure 7 shows another mono-leg TEG concept orientated on classical TEG design. 
a)

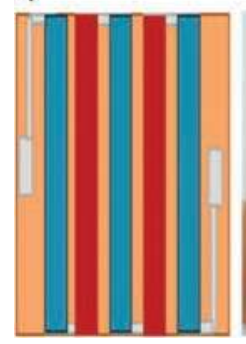

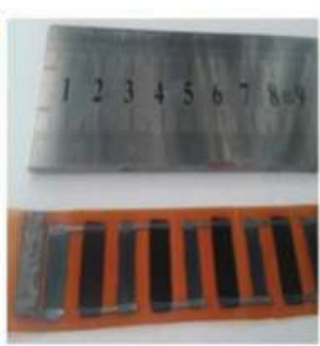

b)

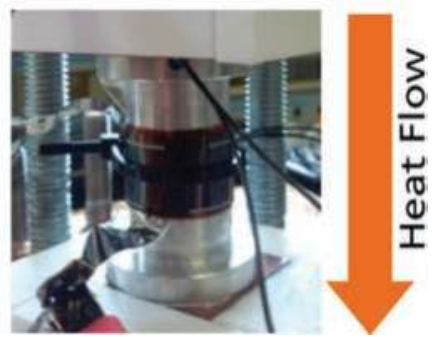

Figure 6. (a) Scheme of a TEG design with p-type (red) and n-type (blue) polymers and the actually printed polymer TEG with PEDOT:PSS and Poly[K $\mathrm{K}_{x}(\mathrm{Ni}$-ett)] on Polyimide (PI) substrate. (b) TEG wrapped around a holder for measuring power output by applying defined temperature difference. The heat flow is parallel to the substrate.

a)

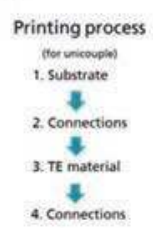

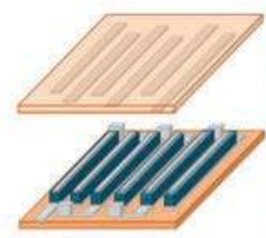

b)

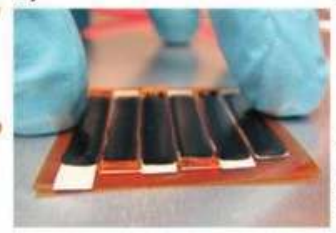

c)

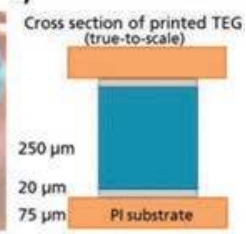

Figure 7. (a) Printing sequence and scheme of a vertical TEG design. (b) Vertical polymer TEG printed with dispenser, substrate is PI, polymer is PEDOT:PSS with $5 \%$ DMSO. (c) Schematic cross section and dimensions of printed vertical TEG.

This design has the advantage consisting in the fact that heat flow goes only thorough the active polymer, preventing parasitic heat flows. Furthermore, fill factor and contact resistances can be reduced by larger contact areas in comparison to the other design. The challenge in printing polymer TEG is, however, that the pastes/ink which are used for printing TEGs have very high amount of solvents, which makes it difficult to quickly build up thick layers. PEDOT:PSS paste used for printing TEG in Figure 7 contained $~ 2$ wt.\% PEDOT:PSS. This ratio of solvent and solid component is necessary for avoiding agglomeration and, thus, for providing stable printable polymer paste. Therefore, printing of thick layers is time consuming, because after deposition of each layer it is necessary to heat up polymer to evaporate solvent. Typical thickness of one layer after removing the solvent is in the range of 10-20 $\mu \mathrm{m}$. Therefore, printing time of TEG shown in Figure 7 was relatively long about 5 hours (in a lab environment).

Thus, polymer TEGs designs and configurations are still not satisfying and need to be improved. Simulations by Oshima et al. [71] based on the work of Aranguren et al. [72] have shown theoretically the possibility to reach power output in the range of MWh/year by polymer TEGs with a new optimized design based on porous substrate. In this publication, relatively high temperatures of $>100^{\circ} \mathrm{C}$ were assumed. However, experimental realization is still missing.

But, potential application of polymer TEG is strongly limited by physical and chemical properties of polymers. Thermal stability of polymers is very critical for applications, as was shown recently by Stepien et al. It was shown for PEDOT:PSS, that electrical conductivity drops irreversibly 
down for temperatures $>55^{\circ} \mathrm{C}$ (further details are given in Subsection 4.2.). It was assumed, that the reason might be degradation of the polymer. This behavior was observed under ambient conditions as well as in glove box under an inert atmosphere. That means, degradation of polymer properties starts at temperatures around $50^{\circ} \mathrm{C}$ and limits possible application dramatically. Thus, investigation by Stepien et al. has shown that the challenge for polymers is, on the one hand, to increase ZT values, but, on the other hand, to increase thermal stability also.

Power output and efficiency of polymer TEG are dependent on both the ZT value and maximum possible temperature difference.

Theoretical expression of the maximum efficiency of TEG is:

$$
\eta_{\max }=\frac{T_{H}-T_{C}}{T_{H}} \frac{\sqrt{1+Z \bar{T}}-1}{\sqrt{1+Z \bar{T}}+\frac{T_{c}}{T_{H}}}
$$

with ZT value for TE module according to Eq. (2):

$$
Z \bar{T}=\frac{\left(S_{p}-S_{n}\right)^{2} \bar{T}}{\left[\left(\rho_{n} \kappa_{n}\right)^{1 / 2}+\left(\rho_{p} \kappa_{p}\right)^{1 / 2}\right]^{2}},
$$

where $T_{C^{\prime}} T_{H^{\prime}}$ and $\bar{T}$ are the temperature at the cold side, hot side, and average ambient absolute temperature, respectively; $S_{n}, S_{p}, \rho_{n}, \rho_{p}, \kappa_{n}$, and $\kappa_{p}$ are Seebeck coefficient, electrical resistivity, and thermal conductivity of $\mathrm{n}$ - and p-type polymers, respectively.

The temperature dependence of TEG efficiency calculated according to Eq. (1) is shown in Figure 8. Calculations performed with ZT values equals 1 [7] and 0.2 [8] for p-type and n-type polymers, respectively, efficiency below $0.25 \%$ can be reached by applying maximum temperature difference of $60 \mathrm{~K}(333-273 \mathrm{~K})$.

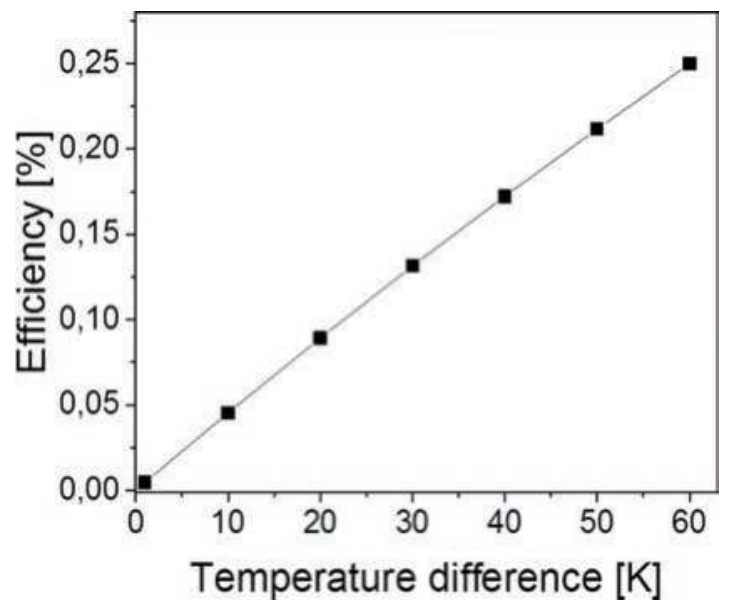

Figure 8. Theoretical temperature dependence of maximum efficiency for polymer TEG with p- and n-type polymers from references $[7,8]$. Cold side temperature equals to $273 \mathrm{~K}$. Internal contact resistance was assumed as ideal. 
Polymer TEGs based on metal organic materials with 35 thermocouples (p- and n-type legs) have delivered around $1 \mu \mathrm{W} / \mathrm{cm}^{2}$ at temperature difference of $25 \mathrm{~K}$ [8]. Another TEG made of PEDOT:TOS in a different design delivered $0.27 \mu \mathrm{W} / \mathrm{cm}^{2}$ at a temperature difference of $30 \mathrm{~K}$.

If ZT value or working temperature difference between hot and cold sides of polymer TEG cannot be increased, then polymer TEG can be used for low power application only. Therefore, there is a big challenge to avoid degradation of polymers at higher temperatures.

Another reason for relatively low power output is high contact resistance between polymer and metal contacts. The measurement of contact resistance between PEDOT:PSS and Silver paste reached $5 \times 10^{-2} \mathrm{Ohm} \times \mathrm{cm}^{2}$. This leads to relatively high internal resistance values for printed polymer TEG. Measured internal resistance of printed TEGs was equal to a few $\mathrm{kOhm}$. Internal resistance of commercial TEG with areas of, for example, $4 \mathrm{~cm} \times 4 \mathrm{~cm}$ is $<10 \mathrm{Ohm}$ and efficiency for energy conversion is indicated around $5 \%$ by TEG suppliers. Conventional thermoelectric modules and materials were developed, of course, over decades. Design of commercial TEG was developed and simulated carefully. Leg-length and leg-cross sections were studied in order to get high fill factor, low internal resistances, and optimized power output. Such optimization processes are still missing for polymer TEG.

Estimated costs per Watt (\$/W) of conventional TEG are in the range of $4 \$ / \mathrm{W}$. More than $50 \%$ of the costs are for system itself and manufacturing and just small part for thermoelectric material itself [73].

On the one hand, printing technology is, of course, advantageous for polymer thermoelectrics, because it has the potential for reducing system costs in general. However, on the other hand, if we compare polymer material costs to, for example, today costs of BiTe-based material, we find a large discrepancy. Assuming that costs for producing $1 \mathrm{~kg}$ BiTe-based material equals to $1000 €$, we have costs for $500 \mathrm{ml}$ of PEDOT:PSS dispersion of $\sim 400 €$ today. Thus, cost for $500 \mathrm{ml}$ PEDOT:PSS or more precisely $1 \mathrm{~kg}$ pure PEDOT:PSS is much higher than that of the BiTe-based material, considering the fact that PEDOT:PSS dispersion is purchased with a solid content of only around $2 \mathrm{wt} \%$. Using cost-saving manufacturing technique like printing is an advantage for polymer thermoelectrics of course; however, this advantage is not the key for economical applications without much lower polymer material costs.

The applications of polymer thermoelectrics seem to be for the medium-long term in the lowpower area. The amortization of TEG costs by energy harvesting with polymer TEG is today still difficult and ambitiously.

First applications for polymer TEGs are imaginable if the flexibility and the low weight of the polymer devices are the criteria for choosing the polymer material for thermoelectrics.

\section{Challenges}

\subsection{Material optimization}

Concerning optimization of thermoelectric material, one advantage over nonpolymer materials is the possibility to decouple partially chemistry from morphology. Indeed, changes in 
chemistry also have effect on the conformation of polymer chains, thus the morphology is affected; however, it can be stated that Seebeck coefficient is mainly governed by chemical factors, for example, the kind of counterions used or degree of oxidation, while electrical conductivity is additionally influenced by morphological aspects like crystal orientation, crystal size, or namely charge carrier mobility. Therefore, it is favorable to optimize these two aspects separately. Therefore, parameters of polymer thermoelectric materials can be improved according to the following concept, which can be applied to almost all thermoelectric polymer materials; here special emphasis is given to PEDOT.

\subsubsection{Maximizing electrical conductivity}

Morphology should be optimized by using a proper deposition technique and/or using cosolvents or other additives. The aim of this step is to maximize electrical conductivity. The solution-sheared deposition technique seems to be a promising approach. In this case, Seebeck coefficient should not be influenced significantly.

\subsubsection{Optimization of Seebeck coefficient}

Continuing theme of high electrically conductive material, increase in Seebeck coefficient with proper reduction/oxidation (deprotonating/protonating) methods will be required to obtain good thermoelectric material.

Reduction can be done by electrochemical methods due to its good control of the degree of oxidation. Reduction can also be done by other methods like dipping or immersion in corresponding agents. The aim here is to find the optimum between electrical conductivity and Seebeck coefficient, because change in charge carrier density will affect Seebeck coefficient and electrical conductivity to the contrary.

It should be emphasized, that possible formation of crystals, because of the reducing agents used, should be avoided in order to maintain the crystal structure prepared in step one. This is also true for the use of composite materials. Composites with beneficial thermoelectric properties contain often high quantity of nanoparticles like nanowires, graphene sheets, or others (often over $50 \mathrm{wt} \%$ ), which as a result is nanoparticle-matrix filled with polymers. For these improvements, it is crucial to have absolutely robust processing techniques, as well as, characterization routines.

If these factors cannot be controlled properly, then poor reproducibility of material parameters will be the result. Since thermoelectric properties of polymers are highly dependent on morphological aspects, it is necessary to have ultimate control of film formation and conformation of polymer chains during this processing step.

Reported results often lack comparability because of different characterization methods. For instance, Seebeck coefficient can vary significantly, when electrodes with different geometries are used during the measurement. It was shown, that variation in electrode geometry can influence Seebeck coefficient by a factor of 3 or more [74]. Regarding electrical conductivity, it is known that different methods, for example, van der Pauw or linear 4-point-probe, can result in different sheet resistances because of possible anisotropies in the samples. The same 
applies for measuring film thickness. Regarding the method used, variations of more than $30 \%$ for films in nanometer range can occur. These systematic uncertainties, as well as, nonsystematic ones, will have significant impact on power factor and, hence, the figure of merit. Figure 9 shows the range of uncertainty for power factor over electrical conductivity.

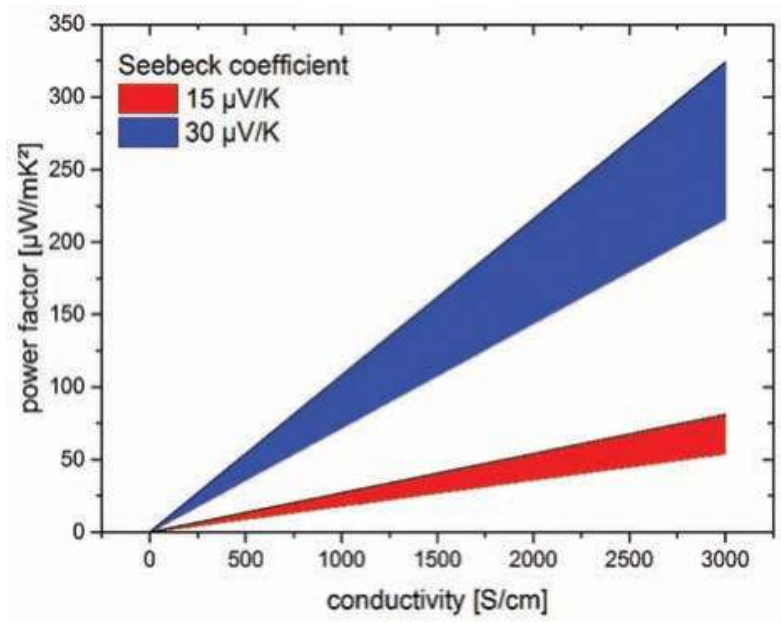

Figure 9. Range of uncertainty for power factor over electrical conductivity. Total uncertainties for Seebeck coefficient and electrical conductivity are $10 \%$ and $5 \%$, respectively.

It can be seen, that overestimated Seebeck coefficient (maybe due to not proper electrode contacts) can lead to dramatic differences. This is especially true for high conducting materials.

Note that more efforts focus recently on measurements of thermal conductivities, as well as, anisotropy of the overall material properties and should be further encouraged.

\subsection{Thermal stability}

Thermoelectric energy conversion is considered to be a robust and sustainable process. This claim can only be fulfilled if material degradation can be avoided. One drawback, compared to other low temperature materials like BiTe, is besides thermoelectric performance, low thermal stability. Usually, glass transition and crystal melting for polymers occurs at much lower temperatures as for BiTe. This can lead to unfavorable change in nano- and microstructure of the polymer material.

In the case of DMSO doped PEDOT:PSS films, it was shown, that during 50 hours of thermal stress at $75^{\circ} \mathrm{C}$ ambient temperature, electrical conductivity decreased irreversibly by $17 \%$ (Figure 10). For higher temperatures, decrease in electrical conductivity was even more pronounced. Significant chemical degradation is found to start between 140 and $160^{\circ} \mathrm{C}$, which is 
highly undesirable for real long-term applications. This leads to the conclusion that thermoelectric generators made of currently most promising p-type polymer PEDOT:PSS should be deployed only for moderate temperatures, which limits possible applications.

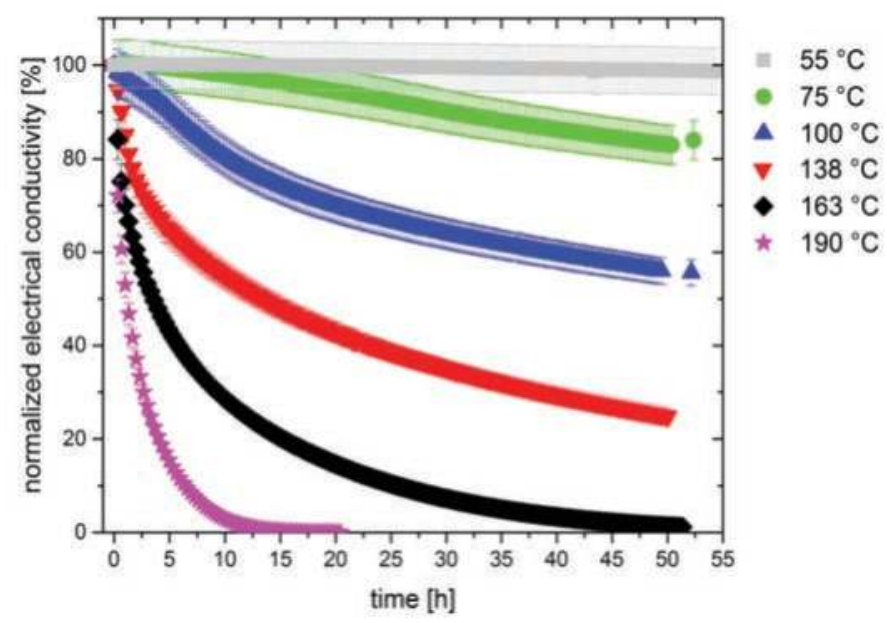

Figure 10. Decrease of normalized electrical conductivity of PEDOT:PSS (+DMSO) films at thermal stress for over 50 hours. Stepien et al. not accepted yet.

Comparing intrinsically conductive polymers with BiTe in terms of thermoelectric performance and thermal stability still a lot of effort has to be put in polymer materials in order to compete with BiTe.

\section{Acknowledgement}

This work has received partial funding from the European Unions' Seventh Framework Programme for research, technological development, and demonstration under the grant agreement No 604647.

\section{Author details}

Lukas Stepien $^{1, *}$, Aljoscha Roch ${ }^{1}$, Roman Tkachov ${ }^{1}$ and Tomasz Gedrange ${ }^{2}$

*Address all correspondence to: lukas.stepien@iws.fraunhofer.de

1 Fraunhofer Institute for Material and Beam Technology IWS, Dresden, Germany

2 University Hospital Carl Gustav Carus at the TU Dresden, Dresden, Germany 


\section{References}

[1] Tsai T-S, Cheng H-C, Chen C-H, Whang W-T.: Widely variable Seebeck coefficient and enhanced thermoelectric power of PEDOT:PSS films by blending thermal decomposable ammonium formate. Organic Electronics. 2012;12(12):2159-2164. DOI: 10.1016/j. orgel.2011.09.004

[2] Toshima N.: Conductive polymers as a new type of thermoelectric material. Macromolecular Symposia. 2002;186(1):81-86 . DOI: 10.1002/1521-3900(200208)

[3] Hiroshige Y, Ookawa M, Toshima N.: Thermoelectric figure-of-merit of iodine-doped copolymer of phenylenevinylene with dialkoxyphenylenevinylene. Synthetic Metals. 2007;157(10-12):467-474. DOI: 10.1016/j.synthmet.2007.05.003

[4] Yan H, Ishida T, Toshima N, China S.: Thermoelectric properties of electrically conductive polypyrrole film. Thermoelectrics, 2001. Proceedings ICT 2001. XX International Conference. 2001;310-313. DOI: 10.1109/ICT.2001.979894

[5] Kim G-H, Shao L, Zhang K, Pipe KP.: Engineered doping of organic semiconductors for enhanced thermoelectric efficiency. Nature Materials. 2013;12:719-723. DOI: 10.1038/ nmat3635

[6] Bubnova O, Khan ZU, Malti A, Braun S, Fahlman M, Berggren M, Crispin X.: Optimization of the thermoelectric figure of merit in the conducting polymer poly(3,4-ethylenedioxythiophene). Nature Materials. 2011;10:429-433. DOI: 10.1038/nmat3012

[7] Park T, Park C, Kim B, Shin H, Kim E.: Flexible PEDOT electrodes with large thermoelectric power factors to generate electricity by the touch of fingertips. Energy \& Environmental Science. 2013;6:788-792. DOI: 10.1039/c3ee23729j

[8] Sun Y, Sheng P, Di C, Jiao F, Xu W, Qiu D, Zhu D.: Organic thermoelectric materials and devices based on p- and n-Type Poly(metal 1,1,2,2-ethenetetrathiolate)s. Advanced Materials. 2012;24:932-937. DOI: 10.1002/adma.201104305

[9] Mengistie DA, Ibrahem MA, Wang P-C, Chu C-W.: Highly conductive PEDOT:PSS treated with formic acid for ITO-Free polymer solar cells. ACS Applied Material Interfaces. 2014;6(4):pp. 2292-2299. DOI: 10.1021/am405024d

[10] Tan L, Zhou H, Ji T, Huang L, Chen Y.: High conductive PEDOT via post-treatment by halobenzoic for high-efficiency ITO-free and transporting layer-free organic solar cells. Organic Electronics. 2016;33:316-323. DOI: 10.1016/j.orgel.2016.03.037

[11] Worfolk BJ, Andrews SC, Park S, Reinspach J, Liu N, Toney MF, Mannsfeld SCB, Bao Z.: Ultrahigh electrical conductivity in solution-sheared polymeric transparent films. PNAS. 2015;112(46):14138-14143. DOI: 10.1073/pnas.1509958112

[12] Gueye MN, Carella A, Massonnet N, Yvenou E, Brenet S, Faure-Vincent J, Pouget S, Rieutord F, Okuno H, Benayad A, Demadrille R, Simonato J-P.: Structure and dopant 
engineering in PEDOT thin films: Practical tools for a dramatic conductivity enhancement. Chemistry of Materials. 2016;28(10):3462-3468. DOI: 10.1021/acs.chemmater.6b01035

[13] Cho B, Park KS, Baek J, Oh HS, Koo Lee Y-E, Sung MM.: Single-Crystal Poly(3,4ethylenedioxythiophene) Nanowires with Ultrahigh Conductivity. Nano Letters. 2014;14(6):3321-3327. DOI: 10.1021/nl500748y

[14] Mateeva N, Niculescu H, Schlenoff J, Testardi LR.: Correlation of Seebeck coefficient and electric conductivity in polyaniline and polypyrrole. Journal of Applied Physics. 1998;83(6):3111-3117 . DOI: 10.1063/1.367119

[15] Li J, Tang X, Li H, Yan Y, Zhang Q.: Synthesis and thermoelectric properties of hydrochloric acid-doped polyaniline. Synthetic Metals. 2010;160(11-12):1153-1158. DOI: 10.1016/j. synthmet.2010.03.001

[16] Feng-Xing J, Jing-Kun X, Bao-Yang L, Yu X, Rong-Jin H, Lai-Feng L.: Thermoelectric performance of Poly(3,4-ethylenedioxythiophene): Poly(styrenesulfonate). Chinese Physics Letters. 2008;25(6):2202-2205.

[17] Atkins P, d'Sousa J. Atkins' Physical Chemistry. 10th ed. Oxford: Oxford University Press; 2014. 1008 p. DOI: ISBN 978-0199697403

[18] Botelho AI, Shin Y, Liu J, Li X.: Structure and optical bandgap relationship of $\pi$-conjugated systems. PLoS One. 2014;9(1):e86370. DOI: 10.1371/journal.pone.0086370

[19] Rehan M.: Elektrisch leitfähige Kunststoffe. Chemie unserer Zeit. 2003;37(1):18-29.

[20] Thyssen Krupp Plastics. Technische Kunststoffe im Überblick [Internet]. Available from: http://www.thyssenkrupp-plastics.de/fileadmin/inhalte/07_Publikationen/06_Prospekte/ 0750_Techn_Kunststoffe_150dpi.pdf [Accessed: 18.08.2016].

[21] Liu J, Wang X, Li D, Coates NE, Segalman RA, Cahill DG.: Thermal conductivity and elastic constants of PEDOT:PSS with high electrical conductivity. Macromolecules. 2015;48:585-591. DOI: 10.1021/ma502099t

[22] Han L, Spangsdorf SH, Nong NV, Hung LT, Zhang YB, Pham HN, Chen YZ, Roch A, Stepien L, Pryds N.: Effects of spark plasma sintering conditions on the anisotropic thermoelectric properties of bismuth antimony telluride. RSC Advances. 2016;6(64):5956559573. DOI: 10.1039/C6RA06688G

[23] Kim SI, Lee KH, Mun HA, Kim HS, Hwang SW, Roh JW, Yang DJ, Shin WH, Li XS, Lee YH, Snyder GJ, Kim SW.: Dense dislocation arrays embedded in grain boundaries for high-performance bulk thermoelectrics. Science. 2015;348(6230):109-114. DOI: 10.1126/ science.aaa4166

[24] MacDiarmid AG, Epstein AJ.: The concept of secondary doping as applied to polyaniline. Synthetic Metals. 1994;65(2-3):103-116. DOI: 10.1016/0379-6779(94)90171-6 
[25] Tsukamoto J, Takahashi A, Kawasaki K.: Structure and electrical properties of polyacetylene yielding a conductivity of $105 \mathrm{~S} / \mathrm{cm}$. Japanese Journal of Applied Physics. 1990;29(1):125-130.

[26] He M, Qiu F, Lin Z.: Towards high-performance polymer-based thermoelectric materials. Energy \& Environmental Science. 2013;6(5):1341-1642. DOI: 10.1039/c3ee24193a

[27] Kamarudin MA, Sahamir SR, Datta RS.: A Review on the Fabrication of PolymerBased Thermoelectric Materials and Fabrication Methods. The Scientific World Journal. 2013;1-17. DOI: 10.1155/2013/713640

[28] Culebras M, Gómez CM, Cantarero A.: Review on polymers for thermoelectric applications. Materials. 2014;7:6701-6732. DOI: 10.3390/ma7096701

[29] Sun K, Zhang S, Li P, Xia Y, Zhang X, Du D, Isikgor FH, Ouyang J.: Review on application of PEDOTs and PEDOT:PSS in energy conversion and storage devices. Journal of Material Sciences. 2015;26:4438-4462. DOI: 10.1007/s10854-015-2895-5

[30] Wei Q, Mukaida M, Kirihara K, Naitoh Y, Ishida T.: Recent progress on PEDOT-based thermoelectric materials. Materials. 2015;8:732-750. DOI: 10.3390/ma8020732

[31] Katz HE, Poehler TO, editors.: Innovative Thermoelectric Materials. 1st ed. London: Imperial College Press; 2016. 292 p. DOI: ISBN 978-1783266050

[32] Russ B, Glaudell A, Urban JJ, Chabinyc ML, Segalman RA.: Organic thermoelectric materials for energy harvesting and temperature control. Nature Reviews Materials.2016;1:16050. DOI: 10.1038/natrevmats.2016.50

[33] Lee SH, Park H, Son W, Choi HH.: Novel solution-processable, dedoped semiconductors for application in thermoelectric devices. Journal of Materials Chemistry A. 2014;2:13380. DOI: $10.1039 / \mathrm{c} 4 \mathrm{ta} 01839 \mathrm{~g}$

[34] Xiong J, Jiang F, Zhou W, Liu C, Xu J.: Highly electrical and thermoelectric properties of a PEDOT:PSS thin-film via direct dilution-filtration. RSC Advances. 2015;5:60708. DOI: $10.1039 / \mathrm{c} 5 \mathrm{ra} 07820 \mathrm{~b}$

[35] Yoo D, Kim J, Kim JH.: Direct synthesis of highly conductive poly(3,4ethylenedioxythiophene):poly(4-styrenesulfonate) (PEDOT:PSS)/graphene composites and their applications in energy harvesting systems. Nano Research. 2014;7(5):717-730. DOI: $10.1007 / \mathrm{s} 12274-014-0433-\mathrm{z}$

[36] Bae EJ, Kang YH, Jang K-S, Cho SY.: Enhancement of thermoelectric properties of PEDOT:PSS and tellurium-PEDOT:PSS hybrid composites by simple chemical treatment. Scientific Reports. 2016;6:18805. DOI: 10.1038/srep18805

[37] Hong CT, Kang YH, Ryu J, Cho SY, Jang K-S.: Spray-printed CNT/P3HT organic thermoelectric films and power generators. Journal of Materials Chemistry A. 2015;3:2142821433. DOI: 10.1039/C5TA06096F 
[38] Qu S, Yao Q, Wang L, Chen Z, Xu K, Zeng H, Shi W, Zhang T, Uher C, Chen L.: Highly anisotropic P3HT films with enhanced thermoelectric performance via organic small molecule epitaxy. NPG Asia Materials. 2016;8:e292. DOI: doi:10.1038/am.2016.97

[39] Jiao F, Di CA, Sun Y, Sheng P, Xu W, Zhu D.: Inkjet-printed flexible organic thin-film thermoelectric devices based on p- and n-type poly(metal 1,1,2,2-ethenetetrathiolate) s/polymer composites through ball-milling. Philosophical Transactions of the Royal Society A: Mathematical, Physical and Engineering Sciences. 2013;10:20130008. DOI: 10.1098/rsta.2013.0008

[40] Zuzok R, Kaiser AB, Pukacki W, Roth S.: Thermoelectric power and conductivity of iodine-doped "new" polyacetylene. Journal of Chemical Physics. 1991;95:1270. DOI: $10.1063 / 1.461107$

[41] Du Y, Shen SZ, Yang W, Donelson R, Cai K, Casey PS.: Simultaneous increase in conductivity and Seebeck coefficient in a polyaniline/graphene nanosheets thermoelectric nanocomposite. Synthetic Metals. 2012;161(23-24):2688-2692. DOI: 10.1016/j. synthmet.2011.09.044

[42] Wang L, Yao Q, Xiao J, Zeng K, Qu S, Shi W, Wang Q, Chen L.: Engineered molecular chain ordering in single-walled carbon nanotubes/polyaniline composite films for highperformance organic thermoelectric materials. Chemistry - An Asian Journal. 216;11(12) DOI: $10.1002 / a s i a .201600212$

[43] Wang L, Yao Q, Bi H, Huang F, Wang Q, Chen L.: PANI/graphene nanocomposite films with high thermoelectric properties by enhanced molecular ordering. Journal of Material Chemistry A. 2015;3:7086-7092. DOI: 10.1039/C4TA06422D

[44] Wang ZH, Ichimura K, Dresselhaus MS, Dresselhaus G, Lee WT, Wang KA, Eklund PC.: Electronic transport properties of KxC70 thin films. Physical Review B. 1993;48(14):1065710660. DOI: 10.1103/PhysRevB.48.10657

[45] Menke T, Ray D, Meiss J, Leo K, Riede M.: In-situ conductivity and Seebeck measurements of highly efficient n-dopants in fullerene C60. Applied Physics Letters. 2012;100:093304. DOI: $10.1063 / 1.3689778$

[46] Schlitz RA, Brunetti FG, Glaudell AM, Miller PL, Brady MA, Takacs CJ, Hawker CJ, Chabinyc ML.: Solubility-limited extrinsic n-Type doping of a high electron mobility polymer for thermoelectric applications. Advanced Materials. 2014;26:2825-2830. DOI: 10.1002/adma.201304866

[47] Russ B, Robb MJ, Brunetti FG, Miller PL, Perry EE, Patel SN, Ho V, Chang WB, Urban JJ, Chabinyc ML, Hawker CJ, Segalman RA.: Power factor enhancement in solution-processed organic n-Type thermoelectrics through molecular design. Advanced Materials. 2014;26:3473-3477. DOI: 10.1002/adma.201306116

[48] Kim SL, Choi K, Tazebay A, Yu C.: Flexible power fabrics made of carbon nanotubes for harvesting thermoelectricity. ACS Nano. 2014;8:2377-2386. DOI: 10.1021/nn405893t 
[49] Shi K, Zhang F, Di C-A, Yan T-W, Zou Y, Zhou X, Zhu D, Wang J-Y, Pei J.: Toward high performance n-type thermoelectric materials by rational modification of BDPPV backbones. Journal of the American Chemical Society. 2015;137:6979-6982. DOI: 10.1021/ jacs.5b00945

[50] Fukumaru T, Fujigaya T, Nakashima N.: Development of n-type cobaltocene-encapsulated carbon nanotubes with remarkable thermoelectric property. Science Reports. 2015;5:7951. DOI: 10.1038/srep07951

[51] Sun Y, Zhang F, Sun Y, Di C, Xu W, Zhu D.: n-Type thermoelectric materials based on CuTCNQ nanocrystals and CuTCNQ nanorod arrays. Journal of Materials Chemistry A. 2015;3:2677. DOI: 10.1039/c4ta06475e

[52] Hu D, Liu Q, Tisdale J, Lei T, Pei J, Wang H, Urbas A, Hu B.: Seebeck Effects in n-Type and p-Type polymers driven simultaneously by surface polarization and entropy differences based on conductor/polymer/conductor thin-film devices. ACS Nano. 2015;9:5208. DOI: $10.1021 /$ acsnano.5b00589

[53] Sanduleac I, Casian A.: Nanostructured TTT(TCNQ)2 organic crystals as promising thermoelectric n-Type materials: 3D modeling . Journal of Electronic Materials. 2016;45:1316-1320. DOI: 10.1007/s11664-015-4018-8

[54] Yoo D, Lee JJ, Park C, Choi HH, Kim JH.: N-type organic thermoelectric materials based on polyaniline doped with the aprotic ionic liquid 1-ethyl-3-methylimidazolium ethyl sulfate. RSC Advances. 2016;6:37130-37135. DOI: 10.1039/c6ra02334g

[55] Sun Y, Qiu L, Tang L, Geng H, Wang H, Zhang F, Huang D, Xu W, Yue P, Guan Y, Jiao F, Sun Y, Tang D, Di C, Yi Y, Zhu D.: Flexible n-Type high-performance thermoelectric thin films of Poly(nickel-ethylenetetrathiolate) prepared by an electrochemical method. Advanced Materials. 2016;28:3351-3358. DOI: 10.1002/adma.201505922

[56] Poleschner H, John W, Hoppe F, Fanghänel E, Roth S.: Synthese und Eigenschaften elektronenleitender Poly-Dithiolenkomplexe mit Ethylentetrathiolat und Tetrathiafulvalentetrathiolat als Bruckenliganden. J. Praktische Chem. 1983;325:957-975. DOI: $10.1002 /$ prac. 19833250612

[57] Holdcroft GE, Underhill AE.: Preparation and electrical conduction properties of polymeric transition metal complexes of 1,1,2,2-ethenetetrathiolate ligand. Synthetic Metals. 1985;10:427. DOI: 10.1016/0379-6779(85)90200-0

[58] Vicente R, Ribas J, Cassoux P, Valade L.: Synthesis, characterization and properties of highly conducting organometallic polymers derived from the ethylene tetrathiolate anion. Synthetic Metals. 1986;13:265. DOI: 10.1016/0379-6779(86)90188-8

[59] Vogt T, Faulmann C, Soules R, Lecante P, Mosset A, Castan P, Cassoux P, Galy J.: A LAXS (Large Angle X-ray Scattering) and EXAFS (Extended X-ray Absorption Fine Structure) investigation of conductive amorphous nickel tetrathiolato polymers. Journal of American Chemical Society. 1988;110:1833-1840. DOI: 10.1021/ja00214a028 
[60] Bellitto C, Bonamico M, Fares V, Imperatori P, Patrizio S.: Tetrathiafulvalenium salts of planar PtII, PdII, and CuII 1,2-dithio-oxalato-S,S' anions. Synthesis, chemistry and molecular structures of bis(tetrathiafulvalenium) bis(1,2-dithio-oxalato-S,S')palladate(II), [ttf]2[Pd(S2C2O2)2], and of bis(tetrathiafulvalenium)tetrathiafulvalene bis(1,2-dithiooxalato-S,S')platinate(II), [ttf]3[Pt(S2C2O2)2]. Journal of the Chemical Society, Dalton Transactions. 1989;719-727. DOI: 10.1039/DT9890000719

[61] Faulmann C, Cassoux P, Vicente R, Ribas J, Jolly CA, Reynolds JR.: Conductive amorphous metal-tetrathiolato polymers: Synthesis of a new precursor C6O2S8 and its derived polymers and laxs structural studies. Synthetic Metals. 1989;29:557-562. DOI: 10.1016/0379-6779(89)90349-4

[62] Yoshioka N, Nishide H, Inagaki K, Tsuchida E.: Electrical conductive and magnetic properties of conjugated tetrathiolate nickel polymers. Polymer Bulletin. 1990;23:631636. DOI: 10.1007/BF01033109

[63] Sarangi R, George SD, Rudd DJ, Szilagyi RK, Ribas X, Rovira C, Almeida M, Hodgson KO, Hedman B, Solomon EI.: Sulfur K-Edge X-ray absorption spectroscopy as a probe of Ligand-Metal bond covalency: Metal vs Ligand oxidation in copper and nickel dithiolene complexes. Journal of the American Chemical Society. 2007;129:2316-2326. DOI: 10.1021/ja0665949

[64] Yamamoto T.: Assignment of pre-edge peaks in K-edge x-ray absorption spectra of 3d transition metal compounds: electric dipole or quadrupole? X-Ray Spectrometry. 2008;37:572-584. DOI: 10.1002/xrs.1103

[65] Massonnet N, Carella A, Jaudouin O, Rannou P, Laval G, Celle C, Simonato J.: Improvement of the Seebeck coefficient of PEDOT:PSS by chemical reduction combined with a novel method for its transfer using free-standing thin films. Journal of Materials Chemistry C. 2014;2:1278-1283. DOI: 10.1039/C3TC31674B

[66] Toshima N, Oshima K, Anno H, Nishinaka T, Ichikawa S, Iwata A, Shiraishi Y.: Novel hybrid organic thermoelectric materials: three-component hybrid films consisting of a nanoparticle polymer complex, carbon nanotubes, and vinyl polymer. Advanced Materials. 2015;27:2246-2251. DOI: 10.1002/adma.201405463

[67] Asano H, Sakura N, Oshima K, Shiraishi Y, Toshima N.: Development of ethenetetrathiolate hybrid thermoelectric materials consisting of cellulose acetate and semiconductor nanomaterials. Japanese Journal of Applied Physics. 2016;55:2S. DOI: 10.7567/JJAP.55.02BB02

[68] Oshima K, Asano H, Shiraishi Y, Toshima N.: Dispersion of carbon nanotubes by poly(Ni-ethenetetrathiolate) for organic thermoelectric hybrid materials. Japanese Journal of Applied Physics. 2016;55:2S. DOI: 10.7567/JJAP.55.02BB07

[69] Jiao F, Di C, Sun Y, Sheng P, Xu W, Zhu D.: Inkjet-printed flexible organic thin-film thermoelectric devices based on $\mathrm{p}$ - and n-type poly(metal 1,1,2,2-ethenetetrathiolate)s/polymer composites through ball-milling. Philosophical Transactions of the Royal Society of London. 2014;372DOI: 10.1098/rsta.2013.0008 
[70] Faulmann C, Chahine J, Jacob K, Coppel Y, Valade L, Caro D.: Nickel ethylene tetrathiolate polymers as nanoparticles: a new synthesis for future applications?. Journal of Nanoparticle Research. 2013;15:1. DOI: 10.1007/s11051-013-1586-5

[71] Oshima K, Shiraishi Y, Toshima N.: Novel nanodispersed polymer complex, Poly(nickel 1,1,2,2-ethenetetrathiolate): preparation and hybridization for n-Type of organic thermoelectric materials. Chemistry Letters. 2015;44:1185-1187. DOI: 10.1246/cl.150328

[72] Aranguren P, Roch A, Stepien L, Abt M, von Lukowicz M, Dani I, Astrain D.: Optimized design for flexible polymer thermoelectric generators. Applied Thermal Engineering. 2016;102:402-411. DOI: 10.1016/j.applthermaleng.2016.03.037

[73] LeBlanc S, Yee SK, Scullin ML, Dames C, Goodson KE.: Material and manufacturing cost considerations for thermoelectrics. Renewable and Sustainable Energy Reviews. 2014;32:313-327. DOI: 10.1016/j.rser.2013.12.030

[74] van Reenen S, Kemerink M.: Correcting for contact geometry in Seebeck coefficient measurements of thin film devices. Organic electronics. 2014;15(10):2250-2255. DOI: 10.1016/j.orgel.2014.06.018 
